Acta Crystallographica Section E

Structure Reports

Online

ISSN 1600-5368

\section{2-Cyanoquinolin-1-ium nitrate}

\section{Wan-Sin Loh, $\neq$ Madhukar Hemamalini and Hoong-Kun Fun*§}

X-ray Crystallography Unit, School of Physics, Universiti Sains Malaysia, 11800 USM, Penang, Malaysia

Correspondence e-mail: hkfun@usm.my

Received 30 September 2010; accepted 30 September 2010

Key indicators: single-crystal X-ray study; $T=100 \mathrm{~K}$; mean $\sigma(\mathrm{C}-\mathrm{C})=0.002 \AA$; $R$ factor $=0.045 ; w R$ factor $=0.122 ;$ data-to-parameter ratio $=16.2$.

A proton is transferred from the nitric acid to the $\mathrm{N}$ atom of 2cyanoquinoline during crystallization, resulting in the formation of the title salt, $\mathrm{C}_{10} \mathrm{H}_{7} \mathrm{~N}_{2}{ }^{+} \cdot \mathrm{NO}_{3}{ }^{-}$. The quinolinium ring system is approximately planar, with a maximum deviation of 0.013 (3) $\AA$. In the crystal, a very asymmetric bifurcated $\mathrm{N}-$ $\mathrm{H} \cdots(\mathrm{O}, \mathrm{O})$ hydrogen bond to two $\mathrm{O}$ atoms of an adjacent nitrate anion occurs, generating an $R_{2}^{1}(4)$ ring motif. C- $\mathrm{H} \cdots \mathrm{O}$ hydrogen bonds link the ions into sheets stacking along the $a$ axis.

\section{Related literature}

For background to and the biological activities of quinoline derivatives, see: Loh, Quah et al. (2010a,b); Loh et al. (2010); Sasaki et al. (1998); Reux et al. (2009); Morimoto et al. (1991); Michael (1997); Markees et al. (1970); Campbell et al. (1988). For the hydrogen-bond motif, see: Bernstein et al. (1995). For related structures, see: Loh, Quah et al. $(2010 a, b)$; Loh et al. (2010). For the stability of the temperature controller used for the data collection, see: Cosier \& Glazer (1986). For bondlength data, see: Allen et al. (1987).

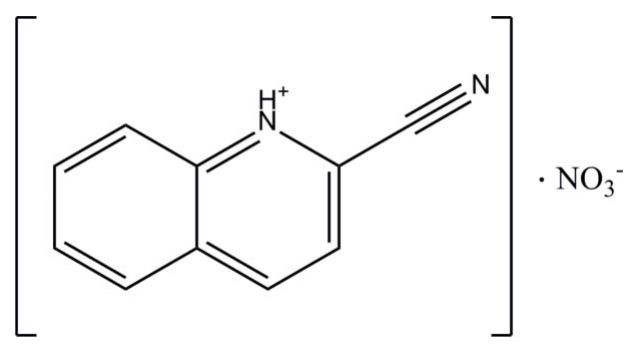

† Thomson Reuters ResearcherID: C-7581-2009. $\S$ Thomson Reuters ResearcherID: A-3561-2009.

\section{Experimental}

$\begin{array}{ll}\text { Crystal data } & \\ \mathrm{C}_{10} \mathrm{H}_{7} \mathrm{~N}_{2}{ }^{+} \cdot \mathrm{NO}_{3}{ }^{-} & V=950.81(3) \AA^{3} \\ M_{r}=217.19 & Z=4 \\ \text { Monoclinic, } P 2_{1} / c & \text { Mo } K \alpha \text { radiation } \\ a=3.6969(1) \AA & \mu=0.12 \mathrm{~mm}^{-1} \\ b=17.7031(3) \AA & T=100 \mathrm{~K} \\ c=14.6029(2) \AA & 0.44 \times 0.18 \times 0.07 \mathrm{~mm}\end{array}$

$\beta=95.802(1)^{\circ}$

Data collection

Bruker SMART APEXII CCD diffractometer

Absorption correction: multi-scan (SADABS; Bruker, 2009)

$T_{\min }=0.951, T_{\max }=0.992$

16184 measured reflections 2758 independent reflections 2103 reflections with $I>2 \sigma(I)$ $R_{\text {int }}=0.041$

Refinement

$R\left[F^{2}>2 \sigma\left(F^{2}\right)\right]=0.045$

$w R\left(F^{2}\right)=0.122$

$S=1.05$

2758 reflections

170 parameters
$\mathrm{H}$ atoms treated by a mixture of independent and constrained refinement
$\Delta \rho_{\max }=0.37$ e $\AA^{-3}$
$\Delta \rho_{\min }=-0.29 \mathrm{e}^{-3}$

Table 1

Hydrogen-bond geometry $\left(\AA{ }^{\circ}\right)$.

\begin{tabular}{lllll}
\hline$D-\mathrm{H} \cdots A$ & $D-\mathrm{H}$ & $\mathrm{H} \cdots A$ & $D \cdots A$ & \multicolumn{1}{l}{$D-\mathrm{H} \cdots A$} \\
\hline $\mathrm{N} 1-\mathrm{H} 1 N 1 \cdots \mathrm{O} 1$ & 0.95 & 2.56 & $3.2376(15)$ & 128 \\
$\mathrm{~N} 1-\mathrm{H} 1 N 1 \cdots \mathrm{O} 3$ & 0.95 & 1.60 & $2.5432(14)$ & 172 \\
$\mathrm{C} 5-\mathrm{H} 5 A \cdots \mathrm{O} 3^{\mathrm{i}}$ & $0.943(16)$ & $2.497(16)$ & $3.2835(16)$ & $141.0(15)$ \\
$\mathrm{C} 7-\mathrm{H} 7 A \cdots \mathrm{O} 2^{\mathrm{ii}}$ & $0.976(16)$ & $2.473(16)$ & $3.3641(16)$ & $151.8(12)$ \\
$\mathrm{C} 8-\mathrm{H} 8 A \cdots \mathrm{O} 2^{\mathrm{iii}}$ & $0.977(18)$ & $2.391(19)$ & $3.3355(17)$ & $162.4(15)$ \\
\hline Symmetry codes: & (i) $-x+1, y+\frac{1}{2},-z+\frac{3}{2} ;$ & (ii) & $-x, y+\frac{1}{2},-z+\frac{3}{2} ;$ & (iii) \\
$x-1,-y+\frac{1}{2}, z-\frac{1}{2}$. & & & &
\end{tabular}

Data collection: APEX2 (Bruker, 2009); cell refinement: SAINT (Bruker, 2009); data reduction: $S A I N T$; $\operatorname{program}(\mathrm{s})$ used to solve structure: SHELXTL (Sheldrick, 2008); program(s) used to refine structure: SHELXTL; molecular graphics: SHELXTL; software used to prepare material for publication: SHELXTL and PLATON (Spek, 2009).

The authors thank Universiti Sains Malaysia (USM) for the Research University Grant (grant No. 1001/PFIZIK/811160). WSL thanks USM for the award of a USM fellowship. MH thanks USM for the award of a postdoctoral fellowship.

Supplementary data and figures for this paper are available from the IUCr electronic archives (Reference: HB5669).

\section{References}

Allen, F. H., Kennard, O., Watson, D. G., Brammer, L., Orpen, A. G. \& Taylor, R. (1987). J. Chem. Soc. Perkin Trans. 2, pp. S1-19.

Bernstein, J., Davis, R. E., Shimoni, L. \& Chang, N.-L. (1995). Angew. Chem. Int. Ed. Engl. 34, 1555-1573.

Bruker (2009). APEX2, SAINT and SADABS. Bruker AXS Inc., Madison, Wisconsin, USA.

Campbell, S. F., Hardstone, J. D. \& Palmer, M. J. (1988). J. Med. Chem. 31, 1031-1035.

Cosier, J. \& Glazer, A. M. (1986). J. Appl. Cryst. 19, 105-107.

Loh, W.-S., Hemamalini, M. \& Fun, H.-K. (2010). Acta Cryst. E66, o2709.

Loh, W.-S., Quah, C. K., Hemamalini, M. \& Fun, H.-K. (2010a). Acta Cryst. E66, o2357. 


\section{organic compounds}

Loh, W.-S., Quah, C. K., Hemamalini, M. \& Fun, H.-K. (2010b). Acta Cryst. E66, o2396.

Markees, D. G., Dewey, V. C. \& Kidder, G. W. (1970). J. Med. Chem. 13, 324326.

Michael, J. P. (1997). Nat. Prod. Rep. 14, 605-608.

Morimoto, Y., Matsuda, F. \& Shirahama, H. (1991). Synlett, 3, 202-203.
Reux, B., Nevalainen, T., Raitio, K. H. \& Koskinen, A. M. P. (2009). Bioorg. Med. Chem. 17, 4441-4447.

Sasaki, K., Tsurumori, A. \& Hirota, T. (1998). J. Chem. Soc. Perkin Trans. 1, pp. $3851-3856$.

Sheldrick, G. M. (2008). Acta Cryst. A64, 112-122.

Spek, A. L. (2009). Acta Cryst. D65, 148-155. 


\section{supporting information}

Acta Cryst. (2010). E66, o2726-o2727 [https://doi.org/10.1107/S1600536810039243]

\section{2-Cyanoquinolin-1-ium nitrate}

\section{Wan-Sin Loh, Madhukar Hemamalini and Hoong-Kun Fun}

\section{S1. Comment}

Recently, hydrogen-bonding patterns involving quinoline and its derivatives with organic acid have been investigated (Loh at al., 2010a,b; Loh et al., 2010). Syntheses of the quinoline derivatives were discussed earlier (Sasaki et al., 1998; Reux et al., 2009). Quinolines and their derivatives are very important compounds because of their wide occurrence in natural products (Morimoto et al., 1991; Michael, 1997) and biologically active compounds (Markees et al., 1970; Campbell et al., 1988). Heterocyclic molecules containing cyano group are useful as drug intermediates. Herein we report the synthesis of 2-cyanoquinolin-1-ium nitrate.

The asymmetric unit of the title compound (Fig. 1) consists of one 2-cyanoquinolin-1-ium cation (C1-C10/N1/N2) and one nitrate anion (N3/O1-O3). One proton is transferred from the hydroxyl group of nitrate to the atom N1 of 2-cyanoquinoline during the crystallization, resulting in the formation of salt. The quinoline ring system $(\mathrm{C} 1-\mathrm{C} 9 / \mathrm{N} 1)$ is approximately planar with a maximum deviation of 0.013 (3) $\AA$ at atom C6. The $R_{2}{ }^{1}(4)$ ring motif (Fig. 1; Bernstein et al., 1995) indicates a bifurcated hydrogen bond from N1-H1N1 to the two acceptors (O1/O3). Bond lengths (Allen et al., 1987) and angles are within the normal ranges and are comparable to the related structures (Loh et al., 2010a,b; Loh et al., 2010).

In the crystal packing (Fig. 2), intermolecular $\mathrm{N} 1-\mathrm{H} 1 \mathrm{~N} 1 \cdots \mathrm{O} 1, \mathrm{~N} 1-\mathrm{H} 1 \mathrm{~N} 1 \cdots \mathrm{O} 3, \mathrm{C} 5-\mathrm{H} 5 \mathrm{~A} \cdots \mathrm{O} 3, \mathrm{C} 7-\mathrm{H} 7 \mathrm{~A} \cdots \mathrm{O} 2$ and $\mathrm{C} 8-\mathrm{H} 8 \mathrm{~A} \cdots \mathrm{O} 2$ hydrogen bonds (Table 1) link the molecules into two-dimensional planes stacking along the $a$ axis.

\section{S2. Experimental}

A few drops of nitric acid were added to a hot methanol solution $(20 \mathrm{ml})$ of quinoline-2-carbonitrile (39 $\mathrm{mg}$, Aldrich) which had been warmed over a magnetic stirrer hotplate for a few minutes. The resulting solution was allowed to cool slowly to room temperature. Colourless plates of (I) appeared after a few days.

\section{S3. Refinement}

All $\mathrm{H}$ atoms were located from a difference Fourier map. H1N1 was fixed at its found position with bond length of $\mathrm{N}-\mathrm{H}$ being $0.9481 \AA$. The remaining $\mathrm{H}$ atoms were refined freely with the bond lengths of $\mathrm{C}-\mathrm{H}$ being 0.943 (17) to 0.998 (17) $\AA$. 


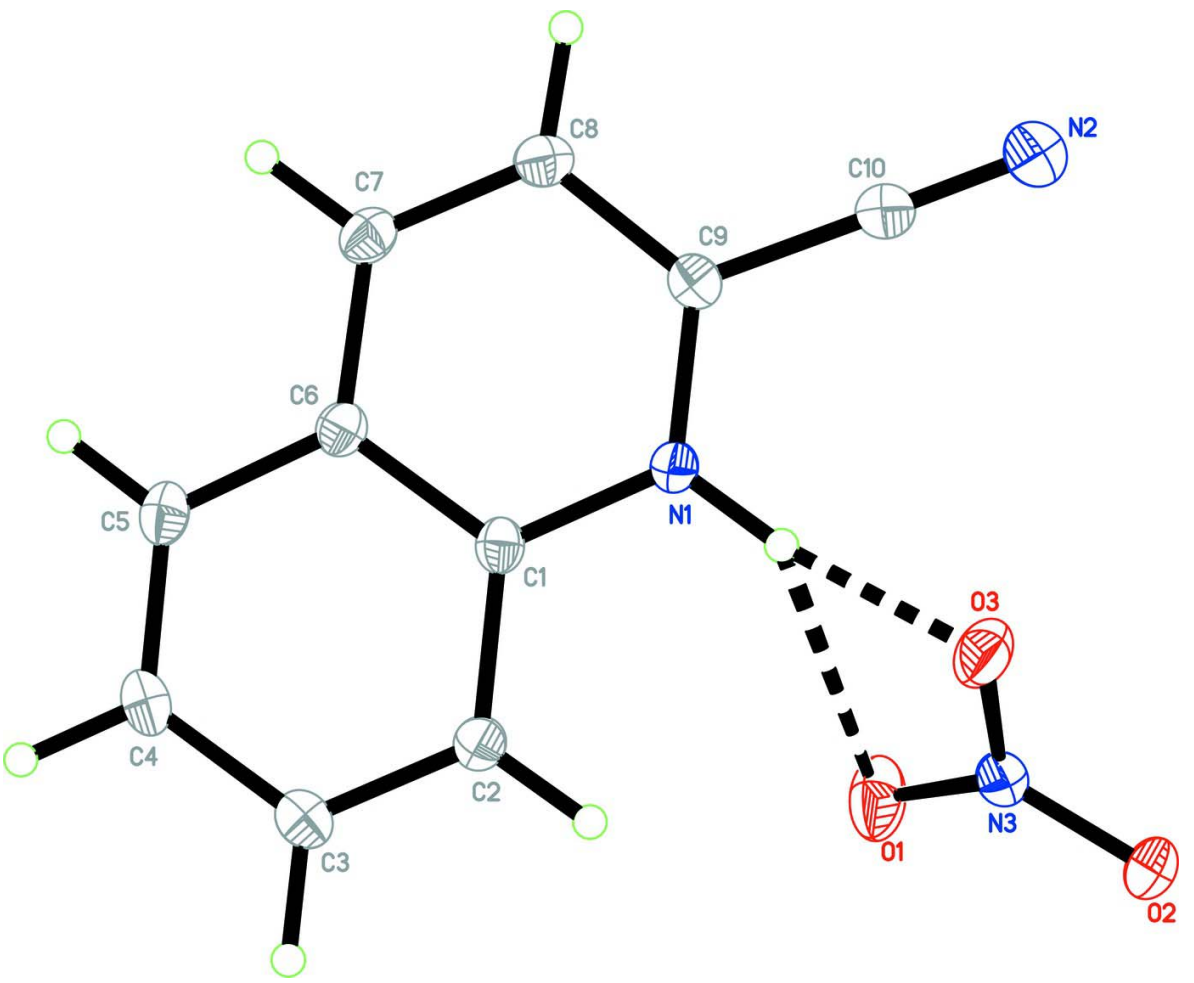

Figure 1

The molecular structure of the title compound showing $50 \%$ probability displacement ellipsoids for non- $\mathrm{H}$ atoms. The $R_{2}{ }^{1}(4)$ ring motif which indicates the bifurcated hydrogen bond is shown.

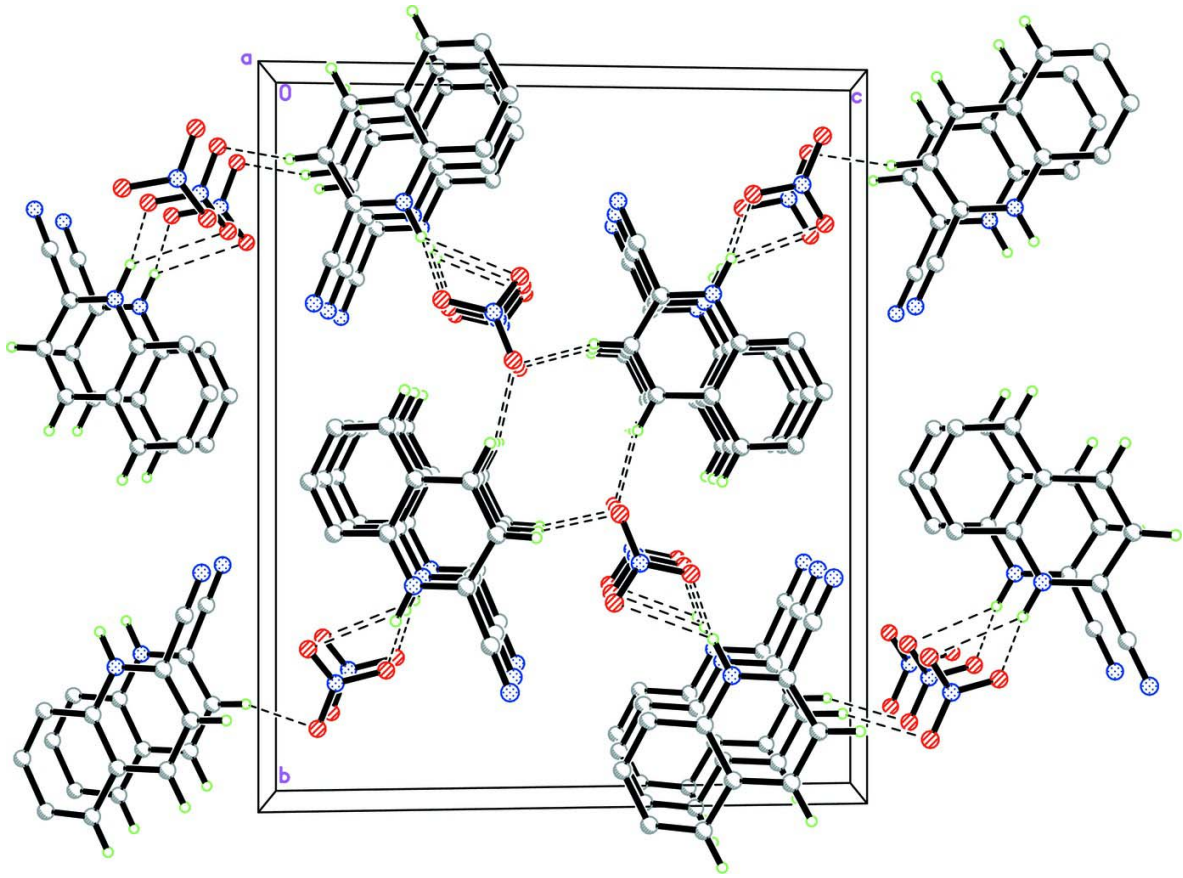

Figure 2

The crystal structure of the title compound, viewed along the $a$ axis. 


\section{2-Cyanoquinolin-1-ium nitrate}

Crystal data

$\mathrm{C}_{10} \mathrm{H}_{7} \mathrm{~N}_{2}{ }^{+} \cdot \mathrm{NO}_{3}{ }^{-}$

$M_{r}=217.19$

Monoclinic, $P 2_{1} / c$

Hall symbol: -P 2 ybc

$a=3.6969$ (1) $\AA$

$b=17.7031$ (3) $\AA$

$c=14.6029$ (2) $\AA$

$\beta=95.802(1)^{\circ}$

$V=950.81(3) \AA^{3}$

$Z=4$

\section{Data collection}

\section{Bruker SMART APEXII CCD}

diffractometer

Radiation source: fine-focus sealed tube

Graphite monochromator

$\varphi$ and $\omega$ scans

Absorption correction: multi-scan

(SADABS; Bruker, 2009)

$T_{\min }=0.951, T_{\max }=0.992$

\section{Refinement}

Refinement on $F^{2}$

Least-squares matrix: full

$R\left[F^{2}>2 \sigma\left(F^{2}\right)\right]=0.045$

$w R\left(F^{2}\right)=0.122$

$S=1.05$

2758 reflections

170 parameters

0 restraints

Primary atom site location: structure-invariant direct methods
$F(000)=448$

$D_{\mathrm{x}}=1.517 \mathrm{Mg} \mathrm{m}^{-3}$

Mo $K \alpha$ radiation, $\lambda=0.71073 \AA$

Cell parameters from 3861 reflections

$\theta=2.7-31.0^{\circ}$

$\mu=0.12 \mathrm{~mm}^{-1}$

$T=100 \mathrm{~K}$

Plate, colourless

$0.44 \times 0.18 \times 0.07 \mathrm{~mm}$

16184 measured reflections

2758 independent reflections

2103 reflections with $I>2 \sigma(I)$

$R_{\text {int }}=0.041$

$\theta_{\max }=30.0^{\circ}, \theta_{\min }=1.8^{\circ}$

$h=-5 \rightarrow 5$

$k=-24 \rightarrow 24$

$l=-20 \rightarrow 20$

Secondary atom site location: difference Fourier map

Hydrogen site location: inferred from neighbouring sites

$\mathrm{H}$ atoms treated by a mixture of independent and constrained refinement

$w=1 /\left[\sigma^{2}\left(F_{\mathrm{o}}^{2}\right)+(0.0614 P)^{2}+0.2227 P\right]$ where $P=\left(F_{\mathrm{o}}^{2}+2 F_{\mathrm{c}}^{2}\right) / 3$

$(\Delta / \sigma)_{\max }<0.001$

$\Delta \rho_{\max }=0.37 \mathrm{e} \AA^{-3}$

$\Delta \rho_{\min }=-0.29$ e $\AA^{-3}$

\section{Special details}

Experimental. The crystal was placed in the cold stream of an Oxford Cryosystems Cobra open-flow nitrogen cryostat (Cosier \& Glazer, 1986) operating at 100.0 (1) K.

Geometry. All e.s.d.'s (except the e.s.d. in the dihedral angle between two 1.s. planes) are estimated using the full covariance matrix. The cell e.s.d.'s are taken into account individually in the estimation of e.s.d.'s in distances, angles and torsion angles; correlations between e.s.d.'s in cell parameters are only used when they are defined by crystal symmetry. An approximate (isotropic) treatment of cell e.s.d.'s is used for estimating e.s.d.'s involving l.s. planes.

Refinement. Refinement of $F^{2}$ against ALL reflections. The weighted $R$-factor $w R$ and goodness of fit $S$ are based on $F^{2}$, conventional $R$-factors $R$ are based on $F$, with $F$ set to zero for negative $F^{2}$. The threshold expression of $F^{2}>\sigma\left(F^{2}\right)$ is used only for calculating $R$-factors $(\mathrm{gt})$ etc. and is not relevant to the choice of reflections for refinement. $R$-factors based on $F^{2}$ are statistically about twice as large as those based on $F$, and $R$-factors based on ALL data will be even larger.

Fractional atomic coordinates and isotropic or equivalent isotropic displacement parameters $\left(\AA^{2}\right)$

\begin{tabular}{lllll}
\hline & $x$ & $y$ & $z$ & $U_{\text {iso }} * / U_{\text {eq }}$ \\
\hline O1 & $0.1851(3)$ & $0.21342(6)$ & $0.92466(7)$ & $0.0314(3)$ \\
O2 & $0.4106(3)$ & $0.10064(5)$ & $0.91484(7)$ & $0.0255(3)$
\end{tabular}




$\begin{array}{lllll}\text { O3 } & 0.4296(3) & 0.17922(5) & 0.80150(6) & 0.0258(3) \\ \mathrm{N} 1 & 0.2524(3) & 0.30942(6) & 0.73844(7) & 0.0144(2) \\ \mathrm{H} 1 \mathrm{~N} 1 & 0.3241 & 0.2630 & 0.7672 & 0.079(8)^{*} \\ \mathrm{~N} 2 & -0.1335(4) & 0.17939(7) & 0.58610(8) & 0.0265(3) \\ \mathrm{N} 3 & 0.3373(3) & 0.16413(6) & 0.88321(7) & 0.0196(3) \\ \mathrm{C} 1 & 0.3582(3) & 0.37566(7) & 0.78076(8) & 0.0142(3) \\ \mathrm{C} 2 & 0.5593(4) & 0.37429(7) & 0.86850(8) & 0.0161(3) \\ \mathrm{C} 3 & 0.6627(4) & 0.44133(7) & 0.90990(9) & 0.0176(3) \\ \mathrm{C} 4 & 0.5697(4) & 0.51140(7) & 0.86660(9) & 0.0181(3) \\ \mathrm{C} 5 & 0.3739(4) & 0.51378(7) & 0.78226(9) & 0.0175(3) \\ \mathrm{C} 6 & 0.2637(3) & 0.44559(7) & 0.73637(8) & 0.0148(3) \\ \mathrm{C} 7 & 0.0675(4) & 0.44419(7) & 0.64838(9) & 0.0176(3) \\ \mathrm{C} 8 & -0.0316(4) & 0.37627(7) & 0.60703(9) & 0.0175(3) \\ \mathrm{C} 9 & 0.0658(4) & 0.30972(7) & 0.65509(8) & 0.0158(3) \\ \mathrm{C} 10 & -0.0406(4) & 0.23670(7) & 0.61661(9) & 0.0184(3) \\ \mathrm{H} 2 \mathrm{~A} & 0.619(4) & 0.3267(9) & 0.8975(11) & 0.020(4)^{*} \\ \mathrm{H} 3 \mathrm{~A} & 0.805(5) & 0.4406(9) & 0.9717(12) & 0.023(4)^{*} \\ \mathrm{H} 4 \mathrm{~A} & 0.649(5) & 0.5595(10) & 0.8975(11) & 0.028(4)^{*} \\ \mathrm{H} 5 \mathrm{~A} & 0.304(5) & 0.5598(9) & 0.7531(11) & 0.024(4)^{*} \\ \mathrm{H} 7 \mathrm{~A} & 0.002(4) & 0.4918(9) & 0.6174(11) & 0.020(4)^{*} \\ \mathrm{H} 8 \mathrm{~A} & -0.166(5) & 0.3735(10) & 0.5461(13) & 0.030(4)^{*}\end{array}$

Atomic displacement parameters $\left(\AA^{2}\right)$

\begin{tabular}{lllllll}
\hline & $U^{11}$ & $U^{22}$ & $U^{33}$ & $U^{12}$ & $U^{13}$ & $U^{23}$ \\
\hline O1 & $0.0486(7)$ & $0.0188(5)$ & $0.0295(6)$ & $0.0018(5)$ & $0.0167(5)$ & $-0.0018(4)$ \\
O2 & $0.0375(6)$ & $0.0142(5)$ & $0.0234(5)$ & $-0.0007(4)$ & $-0.0040(4)$ & $0.0047(4)$ \\
O3 & $0.0438(7)$ & $0.0173(5)$ & $0.0170(5)$ & $0.0075(4)$ & $0.0072(4)$ & $0.0026(4)$ \\
N1 & $0.0169(6)$ & $0.0120(5)$ & $0.0142(5)$ & $0.0006(4)$ & $0.0010(4)$ & $0.0000(4)$ \\
N2 & $0.0331(7)$ & $0.0217(6)$ & $0.0237(6)$ & $-0.0029(5)$ & $-0.0020(5)$ & $-0.0014(5)$ \\
N3 & $0.0255(6)$ & $0.0153(5)$ & $0.0172(5)$ & $-0.0027(4)$ & $-0.0015(4)$ & $-0.0003(4)$ \\
C1 & $0.0146(6)$ & $0.0126(6)$ & $0.0159(6)$ & $0.0002(4)$ & $0.0035(5)$ & $-0.0005(4)$ \\
C2 & $0.0180(6)$ & $0.0145(6)$ & $0.0156(6)$ & $0.0004(5)$ & $0.0013(5)$ & $0.0005(4)$ \\
C3 & $0.0164(6)$ & $0.0187(6)$ & $0.0177(6)$ & $-0.0017(5)$ & $0.0020(5)$ & $-0.0024(5)$ \\
C4 & $0.0180(7)$ & $0.0151(6)$ & $0.0216(6)$ & $-0.0035(5)$ & $0.0044(5)$ & $-0.0037(5)$ \\
C5 & $0.0200(7)$ & $0.0128(6)$ & $0.0204(6)$ & $0.0002(5)$ & $0.0053(5)$ & $0.0002(5)$ \\
C6 & $0.0146(6)$ & $0.0140(6)$ & $0.0160(6)$ & $0.0009(4)$ & $0.0027(5)$ & $0.0008(4)$ \\
C7 & $0.0185(7)$ & $0.0166(6)$ & $0.0177(6)$ & $0.0030(5)$ & $0.0023(5)$ & $0.0023(5)$ \\
C8 & $0.0179(6)$ & $0.0192(6)$ & $0.0153(6)$ & $0.0016(5)$ & $0.0008(5)$ & $0.0009(5)$ \\
C9 & $0.0157(6)$ & $0.0159(6)$ & $0.0158(6)$ & $-0.0003(5)$ & $0.0017(5)$ & $-0.0016(4)$ \\
C10 & $0.0190(7)$ & $0.0192(6)$ & $0.0166(6)$ & $0.0004(5)$ & $0.0003(5)$ & $0.0005(5)$ \\
& & & & & & \\
\hline
\end{tabular}

Geometric parameters $\left(\AA,{ }^{o}\right)$

\begin{tabular}{llll}
\hline $\mathrm{O} 1-\mathrm{N} 3$ & $1.2301(15)$ & $\mathrm{C} 3-\mathrm{H} 3 \mathrm{~A}$ & $0.998(17)$ \\
$\mathrm{O} 2-\mathrm{N} 3$ & $1.2347(14)$ & $\mathrm{C} 4-\mathrm{C} 5$ & $1.3646(19)$ \\
$\mathrm{O} 3-\mathrm{N} 3$ & $1.3015(14)$ & $\mathrm{C} 4-\mathrm{H} 4 \mathrm{~A}$ & $0.993(18)$ \\
$\mathrm{N} 1-\mathrm{C} 9$ & $1.3370(16)$ & $\mathrm{C} 5-\mathrm{C} 6$ & $1.4203(17)$
\end{tabular}




\begin{tabular}{|c|c|c|c|}
\hline $\mathrm{N} 1-\mathrm{C} 1$ & $1.3642(15)$ & $\mathrm{C} 5-\mathrm{H} 5 \mathrm{~A}$ & $0.943(17)$ \\
\hline $\mathrm{N} 1-\mathrm{H} 1 \mathrm{~N} 1$ & 0.9481 & $\mathrm{C} 6-\mathrm{C} 7$ & $1.4104(18)$ \\
\hline $\mathrm{N} 2-\mathrm{C} 10$ & $1.1466(18)$ & $\mathrm{C} 7-\mathrm{C} 8$ & $1.3784(18)$ \\
\hline $\mathrm{C} 1-\mathrm{C} 2$ & $1.4152(17)$ & $\mathrm{C} 7-\mathrm{H} 7 \mathrm{~A}$ & $0.976(17)$ \\
\hline $\mathrm{C} 1-\mathrm{C} 6$ & $1.4243(16)$ & $\mathrm{C} 8-\mathrm{C} 9$ & $1.3998(18)$ \\
\hline $\mathrm{C} 2-\mathrm{C} 3$ & $1.3686(18)$ & $\mathrm{C} 8-\mathrm{H} 8 \mathrm{~A}$ & $0.977(18)$ \\
\hline $\mathrm{C} 2-\mathrm{H} 2 \mathrm{~A}$ & $0.959(16)$ & $\mathrm{C} 9-\mathrm{C} 10$ & $1.4480(18)$ \\
\hline $\mathrm{C} 3-\mathrm{C} 4$ & $1.4188(18)$ & & \\
\hline $\mathrm{C} 9-\mathrm{N} 1-\mathrm{C} 1$ & $120.45(10)$ & $\mathrm{C} 3-\mathrm{C} 4-\mathrm{H} 4 \mathrm{~A}$ & $119.9(10)$ \\
\hline $\mathrm{C} 9-\mathrm{N} 1-\mathrm{H} 1 \mathrm{~N} 1$ & 120.2 & $\mathrm{C} 4-\mathrm{C} 5-\mathrm{C} 6$ & $120.01(12)$ \\
\hline $\mathrm{C} 1-\mathrm{N} 1-\mathrm{H} 1 \mathrm{~N} 1$ & 119.3 & $\mathrm{C} 4-\mathrm{C} 5-\mathrm{H} 5 \mathrm{~A}$ & $122.1(10)$ \\
\hline $\mathrm{O} 1-\mathrm{N} 3-\mathrm{O} 2$ & $123.76(12)$ & $\mathrm{C} 6-\mathrm{C} 5-\mathrm{H} 5 \mathrm{~A}$ & $117.9(10)$ \\
\hline $\mathrm{O} 1-\mathrm{N} 3-\mathrm{O} 3$ & $118.74(11)$ & $\mathrm{C} 7-\mathrm{C} 6-\mathrm{C} 5$ & $122.77(11)$ \\
\hline $\mathrm{O} 2-\mathrm{N} 3-\mathrm{O} 3$ & $117.50(11)$ & $\mathrm{C} 7-\mathrm{C} 6-\mathrm{C} 1$ & $118.63(11)$ \\
\hline $\mathrm{N} 1-\mathrm{C} 1-\mathrm{C} 2$ & $119.72(11)$ & $\mathrm{C} 5-\mathrm{C} 6-\mathrm{C} 1$ & $118.60(12)$ \\
\hline $\mathrm{N} 1-\mathrm{C} 1-\mathrm{C} 6$ & $119.68(11)$ & $\mathrm{C} 8-\mathrm{C} 7-\mathrm{C} 6$ & $120.25(12)$ \\
\hline $\mathrm{C} 2-\mathrm{C} 1-\mathrm{C} 6$ & $120.60(11)$ & $\mathrm{C} 8-\mathrm{C} 7-\mathrm{H} 7 \mathrm{~A}$ & $120.5(10)$ \\
\hline $\mathrm{C} 3-\mathrm{C} 2-\mathrm{C} 1$ & $118.87(12)$ & $\mathrm{C} 6-\mathrm{C} 7-\mathrm{H} 7 \mathrm{~A}$ & $119.2(10)$ \\
\hline $\mathrm{C} 3-\mathrm{C} 2-\mathrm{H} 2 \mathrm{~A}$ & $121.7(10)$ & $\mathrm{C} 7-\mathrm{C} 8-\mathrm{C} 9$ & $118.10(12)$ \\
\hline $\mathrm{C} 1-\mathrm{C} 2-\mathrm{H} 2 \mathrm{~A}$ & $119.4(10)$ & $\mathrm{C} 7-\mathrm{C} 8-\mathrm{H} 8 \mathrm{~A}$ & $122.1(10)$ \\
\hline $\mathrm{C} 2-\mathrm{C} 3-\mathrm{C} 4$ & $121.13(12)$ & $\mathrm{C} 9-\mathrm{C} 8-\mathrm{H} 8 \mathrm{~A}$ & $119.8(10)$ \\
\hline $\mathrm{C} 2-\mathrm{C} 3-\mathrm{H} 3 \mathrm{~A}$ & $119.1(9)$ & $\mathrm{N} 1-\mathrm{C} 9-\mathrm{C} 8$ & $122.87(11)$ \\
\hline $\mathrm{C} 4-\mathrm{C} 3-\mathrm{H} 3 \mathrm{~A}$ & $119.8(9)$ & $\mathrm{N} 1-\mathrm{C} 9-\mathrm{C} 10$ & $116.40(11)$ \\
\hline $\mathrm{C} 5-\mathrm{C} 4-\mathrm{C} 3$ & $120.79(12)$ & $\mathrm{C} 8-\mathrm{C} 9-\mathrm{C} 10$ & $120.72(12)$ \\
\hline $\mathrm{C} 5-\mathrm{C} 4-\mathrm{H} 4 \mathrm{~A}$ & $119.3(10)$ & $\mathrm{N} 2-\mathrm{C} 10-\mathrm{C} 9$ & $178.33(15)$ \\
\hline $\mathrm{C} 9-\mathrm{N} 1-\mathrm{C} 1-\mathrm{C} 2$ & $-179.23(11)$ & $\mathrm{C} 2-\mathrm{C} 1-\mathrm{C} 6-\mathrm{C} 7$ & $179.11(11)$ \\
\hline $\mathrm{C} 9-\mathrm{N} 1-\mathrm{C} 1-\mathrm{C} 6$ & $1.04(18)$ & $\mathrm{N} 1-\mathrm{C} 1-\mathrm{C} 6-\mathrm{C} 5$ & $179.37(11)$ \\
\hline $\mathrm{N} 1-\mathrm{C} 1-\mathrm{C} 2-\mathrm{C} 3$ & $-179.94(12)$ & $\mathrm{C} 2-\mathrm{C} 1-\mathrm{C} 6-\mathrm{C} 5$ & $-0.36(18)$ \\
\hline $\mathrm{C} 6-\mathrm{C} 1-\mathrm{C} 2-\mathrm{C} 3$ & $-0.21(19)$ & $\mathrm{C} 5-\mathrm{C} 6-\mathrm{C} 7-\mathrm{C} 8$ & $179.86(12)$ \\
\hline $\mathrm{C} 1-\mathrm{C} 2-\mathrm{C} 3-\mathrm{C} 4$ & $0.39(19)$ & $\mathrm{C} 1-\mathrm{C} 6-\mathrm{C} 7-\mathrm{C} 8$ & $0.40(19)$ \\
\hline $\mathrm{C} 2-\mathrm{C} 3-\mathrm{C} 4-\mathrm{C} 5$ & $0.0(2)$ & $\mathrm{C} 6-\mathrm{C} 7-\mathrm{C} 8-\mathrm{C} 9$ & $0.44(19)$ \\
\hline $\mathrm{C} 3-\mathrm{C} 4-\mathrm{C} 5-\mathrm{C} 6$ & $-0.6(2)$ & $\mathrm{C} 1-\mathrm{N} 1-\mathrm{C} 9-\mathrm{C} 8$ & $-0.16(19)$ \\
\hline $\mathrm{C} 4-\mathrm{C} 5-\mathrm{C} 6-\mathrm{C} 7$ & $-178.69(12)$ & $\mathrm{C} 1-\mathrm{N} 1-\mathrm{C} 9-\mathrm{C} 10$ & $-178.81(11)$ \\
\hline $\mathrm{C} 4-\mathrm{C} 5-\mathrm{C} 6-\mathrm{C} 1$ & $0.76(19)$ & $\mathrm{C} 7-\mathrm{C} 8-\mathrm{C} 9-\mathrm{N} 1$ & $-0.6(2)$ \\
\hline $\mathrm{N} 1-\mathrm{C} 1-\mathrm{C} 6-\mathrm{C} 7$ & $-1.15(18)$ & $\mathrm{C} 7-\mathrm{C} 8-\mathrm{C} 9-\mathrm{C} 10$ & $177.99(12)$ \\
\hline
\end{tabular}

Hydrogen-bond geometry $\left(A,{ }^{\circ}\right)$

\begin{tabular}{lllll}
\hline$D-\mathrm{H} \cdots A$ & $D-\mathrm{H}$ & $\mathrm{H} \cdots A$ & $D \cdots A$ & $D-\mathrm{H} \cdots A$ \\
\hline $\mathrm{N} 1-\mathrm{H} 1 N 1 \cdots \mathrm{O} 1$ & 0.95 & 2.56 & $3.2376(15)$ & 128 \\
$\mathrm{~N} 1-\mathrm{H} 1 N 1 \cdots \mathrm{O} 3$ & 0.95 & 1.60 & $2.5432(14)$ & 172 \\
$\mathrm{C} 5-\mathrm{H} 5 A \cdots \mathrm{O} 3^{\mathrm{i}}$ & $0.943(16)$ & $2.497(16)$ & $3.2835(16)$ & $141.0(15)$ \\
$\mathrm{C} 7-\mathrm{H} 7 A \cdots \mathrm{O} 2^{\mathrm{ii}}$ & $0.976(16)$ & $2.473(16)$ & $3.3641(16)$ & $151.8(12)$ \\
$\mathrm{C} 8-\mathrm{H} 8 A \cdots \mathrm{O} 2^{\mathrm{iii}}$ & $0.977(18)$ & $2.391(19)$ & $3.3355(17)$ & $162.4(15)$
\end{tabular}

Symmetry codes: (i) $-x+1, y+1 / 2,-z+3 / 2$; (ii) $-x, y+1 / 2,-z+3 / 2$; (iii) $x-1,-y+1 / 2, z-1 / 2$. 